\title{
Gain Tuning of Flight Control Laws for Satisfying Trajectory Tracking Requirements
}

\author{
Urbano Tancredi ${ }^{1}$ and Federico Corraro ${ }^{2}$ \\ 1 University of Naples Parthenope \\ Italian Aerospace Research Centre
}

Italy

\section{Introduction}

The present chapter is concerned with presenting an approach for the synthesis of a gainscheduled flight control law that assures compliance to trajectory tracking requirements. More precisely, a strategy is proposed for improving the tracking performances of a baseline controller, obtained by conventional synthesis techniques, by tuning its gains. The approach is specifically designed for atmospheric re-entry applications, in which gain scheduled flight control laws are typically used.

Gain-scheduling design approaches conventionally construct a nonlinear controller by combining the members of an appropriate family of linear time-invariant (LTI) controllers (Leith \& Leithead, 2000). The time-invariant feedback laws usually share the same structure, and differ only for the values of some tunable parameters, most notably the controller's gains. These gains are generally determined taking advantage of well-assessed LTI-based design techniques, such as pole placement and gain/phase margin methods. However, once a set of LTI feedback laws is specified, the nonlinear controller must be synthesized, which requires an additional design step. This step is of considerable importance since the choice of nonlinear controller realization can greatly influence the closed loop performance (Leith \& Leithead, 2000). Furthermore, actual mission requirements constraint quantitatively the time response of the augmented system (Crespo et al., 2010), e.g. by imposing tracking requirements of a reference trajectory or requiring relevant output variables to be enclosed within a limited flight envelope. As such, the final gain-scheduled controller's performances are ascertained by means of numerical simulation based methods, most notably Monte Carlo, which can highlight limitations that were not apparent in the LTI design phase. As a result, in these cases one is forced to iterate the LTI design, but using analysis results that refer to the nonlinear controller rather than to the LTI ones, further complicating the design improvement task.

Several methods have been proposed in the open literature both for taking into account explicitly the complex dependency of the final controller response from its gains and for dealing with quantitative performance requirements, such as tracking errors. Most, if not all, proposed approaches formulate the design task as an optimization problem, in which the merit function evaluation requires numerical simulation of the augmented system's timeresponse. For instance, (Crespo et al., 2008) develops optimization-based strategies for 
control analysis and tuning at the control verification stage, which build upon numerical evaluation of controller's performance metrics that require simulation of the augmented model. Other authors (dos Santos Coelho, 2009) suggest using chaotic optimization algorithms for enhancing the computational efficiency of the numerical optimization problem. In (Wang \& Stengel, 2002), a robust control law is synthesized using probabilistic robustness techniques, by minimizing a cost that is a function of the probabilities that design criteria will not be satisfied. Monte Carlo simulation is used to estimate the likelihood of system instability and violation of performance requirements subject to variations of the probabilistic system parameters. Stochastic parameter tuning is also proposed in (Miyazawa \& Motoda, 2001), which is a form of optimization by which the probability of the total mission achievement is maximized w.r.t the flight control system's tunable parameters. Mission achievement probability is estimated by applying the Monte Carlo method also in this case.

In this chapter, we propose a methodology for determining all combinations, within a given domain, of the flight control law tunable gains that comply with quantitative requirements expressed in the time domain, and is applicable to nonlinear control laws such as gainscheduled flight control ones. This approach aims at providing quantitative indications on the Flight Control Law (FCL) time-domain performance, taking explicitly into account the complex dependency given by the scheduling of the LTI control laws. As such, it is intended to complement the conventional LTI-based controller synthesis approaches, such as pole/placement and frequency domain methods, which are thus still in charge of addressing the system's stability and robustness.

The approach is based on a technique developed by the authors for tackling a different problem, namely the robustness analysis of a given flight control law (Tancredi et al., 2009). It builds upon a Practical Stability criterion, in which the allowable trajectories dispersion can be specified in the time-domain, in an extremely appealing manner to enforce practical engineering requirements. Under the assumption that the gains domain is a convex polytope, the method results allow distinguishing in the whole domain the gain combinations matching the criterion from those yielding unsatisfactory performance. This is done inferring the nonlinear augmented system behaviour for all gains ranging in a convex polytope from numerical simulations of the augmented dynamics at a limited number of specific points of the gains domain. A set inversion algorithm selects these points using an adaptive gridding strategy. The proposed technique is applied to a gain-scheduled flight control law of the Unmanned Space Vehicle, a re-entry technology demonstrator pursued by the Italian Aerospace Research Centre. Results demonstrate the method's effectiveness in determining the gains combinations allowing to satisfy pre-specified trajectory tracking requirements. Results also show that it is computationally viable and that it allows gaining insight into the factors that limit the controller's performance, thus aiding eventual additional LTI-based design iterations.

\section{Problem setting}

We refer in this work to atmospheric re-entry applications, and to a FCL whose gains are scheduled depending on the values of some specifically selected independent variables, either being a univocal function of the system dynamical state vector, such as air-relative velocity, altitude, Mach number and so on, or explicitly dependent on time. Selection of the 
scheduling law and of the independent variables is out of the scope of the present chapter, because it typically involves exploiting the peculiar flight mechanics features of the application at hand. We assume henceforth that the FCL structure is known, and that the FCL is completely specified once a limited number of parameters, i.e. the FCL gains, are set to a constant value. As introduced in the previous section, the problem dealt with in this chapter is to determine the values of these gains that allow complying to trajectory tracking requirements. Let us assume to have a starting design point that specifies a set of gain values, which typically does not allow satisfying the tracking requirements. We denote this initial guess as the nominal gain value, which is taken equal to zero to simplify notation. Let us also assume to have a finite number $p$ of constant gains and that the gains are enclosed in a bounded set $\Pi \subseteq \mathfrak{R}$, which represents the region in the gains space one wishes to analyze.

The dynamical system we refer to shall be suitable to represent the closed-loop augmented dynamics of an atmospheric re-entry vehicle. The typical FCL for this application foresee a gain-scheduled inner-loop PID control scheme coupled with a time-varying guidance law, possibly dependent on the system state as well. Gain scheduling is taken into account by dependency on the state variables (and time if needed), and the PID action by dependencies on the state, on its time integral (which adds up to the open-loop system's state) and derivative, respectively. Thus, let us consider the following dynamical system, in which $x \in \mathfrak{R}^{n}, y \in \mathfrak{R}^{w}$, and the feedback action is included in the $f(\cdot)$ and $g(\cdot)$ functions.

$$
\dot{x}=f(t, x, \pi) \quad y=g(t, x, \pi)
$$

In the case of an un-powered re-entry vehicle in steep gliding flight, due to the lack of stationary equilibrium solutions for Eq. (1), we refer to time-varying nominal trajectories rather than stationary operating conditions. In addition, these trajectories are usually defined on a finite-time domain, i.e. $t \in[0, T]$, where the initial epoch is taken equal to zero for simplicity and $T$ is a finite positive real number. The nominal trajectory is thus time varying on a compact time domain, and satisfies the following equations.

$$
\begin{gathered}
\tilde{x}(t):=\tilde{x}_{0}+\int_{0}^{t} f(\tau, \tilde{x}, 0) \times d \tau \quad \forall t \in[0, T] \\
\tilde{y}(t):=g(t, \tilde{x}, 0) \quad \forall t \in[0, T]
\end{gathered}
$$

For gain-scheduled FCL, the current design practice relies on the well-known theory of Linear Time Invariant (LTI) systems. In this approach, the original nonlinear system representing the augmented vehicle dynamics is linearized around a limited number of representative time-varying trajectories, including the nominal one. Then, the well-known frozen-time approach is applied (Lee \& Choi, 2004), yielding multiple LTI models. In this way classical design techniques, such as pole placement and gain/phase margin methods, can be exploited. Even if the flight experience has demonstrated that this approach is indeed operative, it is also widely recognized as inefficient (Leith \& Leithead, 2000). In fact, LTIbased analysis may call for gain design values for counteracting poor closed-loop dynamic performances (for instance, low damping or even instabilities) in some of the chosen points on the trajectories. However, the effect of undesirable frozen-time performances on the overall mission objectives can be of scarce importance since the vehicle remains in a particular frozen time condition only for a limited amount of time. Thus, modification of the 
FCL for improving the LTI-based dynamic performances could be un-necessary, since these missions typically specify time-domain criteria, such as nominal trajectory tracking performances, which can be satisfied also in presence of poor frozen-time dynamic performances. LTI-based analysis results are thus usually complemented by dedicated numerical-simulation based analyses, such as Monte Carlo techniques, through which the quantitative dispersion about the reference trajectory can be estimated. Finally, in the LTIbased approach, the gain tuning problem shall be solved in each frozen operating condition, thus considerably limiting the dimension of manageable problems.

The criterion proposed in the present work is instead based on the Practical Stability and/or Finite-Time Stability concepts, whose detailed description can be found in (Gruyitch et al., 2000; Dorato, 2006). This type of stability requires only the inclusion of the system trajectories in a pre-specified subset of the state space, possibly time-varying, in face of bounded initial state displacements and disturbances. As opposed to the classical Lyapunov stability concept it does not require the existence of any equilibrium point, and is independent from Lyapunov stability, in the sense that one neither implies nor excludes the other. The practical stability criterion is inherently well suited to the applications of interest: it allows to take explicitly into account system (1) time domain finiteness, and to use criteria directly linked to the original mission or system requirements, which are typically expressed in terms of trajectory tracking performances. Indeed, the latter can be easily enforced by requiring the inclusion of the system trajectories in a pre-specified time-varying subset of the state space determined by the tracking requirements, to which we refer as the admissible solutions tube, $S_{A}(t)$.

Let us assume the initial state to be perfectly known and equal to the nominal one. In other words, the perturbed output trajectory $y(t ; \pi)$ is defined as a trajectory of system (1) that starts at $t=0$ in $y(0)=y_{0}$, under the constant input $\pi$. This assumption does not limit the scope of the problem, since initial state dispersions can be included, if necessary, as additional elements of the $\Pi$ vector with no conceptual modifications. The tracking requirements are used to define a Boolean property $P$ depending on the gains, so that the system complies with the practical stability criterion if and only if the property is true. In order to gain generality in the capability to enforce admissible dispersion requirements, $P$ is defined in terms of the output trajectories of system (1) (that cover the case in which the system state is analyzed by letting $y=x$ ).

$$
P(\pi)= \begin{cases}\text { true } & y(t ; \pi) \in S_{A}(t) \forall t \in[0, T] \\ \text { false } & \exists t \in[0, T]: y(t ; \pi) \notin S_{A}(t)\end{cases}
$$

The capability of identifying all the combinations of the gain values in $\Pi$ for which the property is true can greatly aid the refinement of the candidate FCL design. Indeed, it allows analyzing the FCL performance over the whole gain domain, as opposed to classical analysis that identifies only a limited number of points in $\Pi$. This aids the FCL upgrade by simplifying the physical understanding of the causes for poor performance. This feature is highly desirable in a design context, in which rather than the knowledge of a certain requirement violation is the determination of the causes that mainly contributes to identify possible design refinement strategies. With this in mind, the gain tuning task is stated as 
determining the set $\Pi_{A}$, subset of $\Pi$, which is made of all the admissible gains, that is, all gains satisfying the tracking requirements.

$$
\Pi_{A}:=\{\pi \in \Pi \mid P(\pi)=\text { true }\}
$$

In this setting, the gain tuning task can be re-formulated as a practical stability analysis problem, as follows.

Problem 1. Given system (1), a bounded set $\Pi \subseteq \mathfrak{R}^{p}$ such that $\pi \in \Pi$, a time-varying compact set $S_{A}(t)$ (admissible solutions tube), and the property $P$, determine the set $\Pi_{A}$.

\section{Solution approach}

In order to simplify the solution to problem 1, we introduce the following restricting assumptions.

Assumption 1. The functions $f(\cdot)$ and $g(\cdot)$ are differentiable in $t, x$ and $\pi$ over relevant domains.

Assumption 2. The gains range in a $p$-dimensional hyper-rectangle $\Pi$.

$$
\Pi:=\left[\underline{\pi}_{1}, \bar{\pi}_{1}\right] \times . . \times\left[\underline{\pi}_{p}, \bar{\pi}_{p}\right]
$$

Assumption 3. The required solutions tube is a w-dimensional hyper-rectangle for all $t \in[0, T]$

$$
S_{A}(t):=\left[\underline{S}_{1 A}(t), \bar{S}_{1 A}(t)\right] \times . . \times\left[\underline{S}_{w A}(t), \bar{S}_{w A}(t)\right]
$$

Various techniques exist being able to deal with the practical stability analysis of a nonlinear dynamical system (see Dorato, 2006, for a survey). The prominent approaches are based on a Lyapunov-type analysis involving an auxiliary function referred to as a Lyapunov-like function in (Gruyitch et al., 2000; Dorato, 2006). However, to the authors' knowledge, there are no systematic and operative means to find a suitable Lyapunov function when nonlinear time-varying systems are considered; Lyapunov-based methods are also inherently conservative in estimating the trajectories dispersion, depending on the selected Lyapunovlike function. A different approach is presented in (Ryali \& Moudgalya, 2005), which stems from the notion of positively invariant tubes. However, it does not bound nor estimates the results conservativeness, with a resulting limited applicability to problems of practical interest. Finally, for Linear Time-Varying (LTV) systems, practical stability analysis approaches have been developed based on operator theory (Amato et al., 2003), which yield only sufficient conditions in the form of a nonlinear, time-varying, differential matrix inequality. Generally speaking, in spite of a wide literature on practical stability theoretical results, all the reported approaches suffer of significant drawbacks when considered from an applicability perspective, including cases where the system dynamics are linear. Indeed, the abundance of theoretical results on practical stability analysis methods it is not balanced by examples of their application to cases of practical engineering interest within the robustness analysis context. 
The approach followed in this chapter extends the one proposed in (Tancredi et al., 2009). for analyzing the robustness of a given flight control law. By setting up the gain tuning task as in Problem 1, this approach can be adapted for being used with the problem at hand with only minor modifications. An overview of the method is repeated in this chapter closely following the one in (Tancredi et al., 2009), but providing additional details and adapting it for dealing with a gain tuning problem. The technique approximates the solution of the practical stability analysis problem for a complex system with the solutions obtained for simpler systems, for which an efficient solution approach can be found. Specifically, the proposed solution approach foresees two successive phases. First, the nonlinear vehicle dynamics are approximated within a pre-specified error tolerance by their time-varying linearizations under several off-nominal gains (approximation phase). Then, problem 1 is solved on the LTV systems obtained in the previous phase taking explicitly into account the approximation error. This is done performing numerical simulations only at suitably selected gains combinations and exploiting the convexity preservation property of the LTV dynamics (property clearance phase). For the sake of clarity, we will describe separately these two phases.

\subsection{Approximation}

Let us consider a partition $\left\{\Pi_{k}\right\}$ of the gain domain, made of hyper-rectangular blocks $\Pi_{k}$, that is, a collection of subsets (blocks) that are both collectively exhaustive and mutually exclusive with respect to the set being partitioned. We then define a collection of LTV systems, each one approximating the nonlinear system in a single block. In particular, each LTV system is obtained linearizing the system around its trajectory obtained by setting the uncertainties to $\pi_{k}^{0}$, the geometrical centre of $\Pi_{k}$. The dynamic equations for each one of such LTV systems as $\pi$ ranges in the relevant $\Pi_{k}$ can be written as:

$$
\begin{aligned}
& \dot{x}_{L k}=\dot{x}_{k}^{0}+A_{k} \times\left(x_{L k}-x_{k}^{0}\right)+G_{k} \times\left(\pi-\pi_{k}^{0}\right) \\
& y_{L k}=y_{k}^{0}+C_{k} \times\left(x_{L k}-x_{k}^{0}\right)+D_{k} \times\left(\pi-\pi_{k}^{0}\right)
\end{aligned}
$$

where the $A_{k}, G_{k}, C_{k}$, and $D_{k}$ matrixes are obtained applying first order expansion of the nonlinear functions in Eq. (1) around $x_{k}^{0}, \Pi_{k}^{0}$. Note that, being the nonlinear system's trajectories time-varying, the centre trajectory and the matrices in Eq. (7) are in general timevarying as well.

In order to quantify the error made in approximating the nonlinear system with the LTV one we use the weighted $L_{\infty}$ norm distance between the nonlinear and linear trajectories, that is, for each LTV system, and thus for each block $\Pi_{k}$ of the partition, we define an approximation error function $e_{k}: \Pi_{k} \rightarrow \mathfrak{R}_{+}$as

$$
e_{k}(\pi):=\left\|y(t ; \pi)-y_{L k}(t ; \pi)\right\|_{\infty}^{b}
$$

We search for an approximation of the nonlinear system that introduces a pre-specified bounded error. Equivalently, this can be seen as searching for a partition $\left\{\Pi_{k}\right\}_{L}$ in which $e_{k}(\cdot)$ is below a pre-specified tolerance $\varepsilon$ for all $\Pi$ in $\Pi$ : 


$$
\left\{\Pi_{k}\right\}_{L}: \forall \Pi_{k} \in\left\{\Pi_{k}\right\}_{L} \quad \max _{\pi \in \Pi k} e_{k}(\pi) \leq \varepsilon
$$

As we will discuss later on, finding such a partition allows using the solution to problem 1 obtained for the LTV systems to approximate the one of the nonlinear system. Assumption 1 assures that a partition complying to Eq. (9) may always be found. Indeed, for any $\Pi_{k}^{0} \in \Pi_{k}$ we have:

$$
\lim _{\pi \rightarrow \pi_{k}^{0}} e_{k}(\pi)=0
$$

Thus, by using a partition of $\Pi$ with sufficiently small blocks, it is possible to approximate as closely as desired the nonlinear trajectories using the LTV ones. Following this fact, an algorithm for finding $\left\{\Pi_{k}\right\}_{L}$ may be obtained by repeatedly shrinking the blocks of the partition for which the approximation error is higher than $\varepsilon$. The partition refinement is here obtained iteratively, by means of an isotropic bisection technique. The isotropic bisection procedure splits a single $p$-dimensional hyper-rectangle set in $2^{p}$ hyper-rectangular subsets, collectively exhaustive and mutually exclusive with respect to the "father" set. These "sons" are generated bisecting in each of the $p$ dimensions the father hyper-rectangle's edges. At each iteration, the approximation error in each block $\Pi_{\mathrm{k}}$ is analyzed. Three cases are possible:

1. $\max _{\pi \in \Pi k} e_{k}(\pi) \leq \varepsilon$. The error is below the tolerance. $\Pi_{k}$ is assigned to $\left\{\Pi_{k}\right\}_{L}:\left\{\Pi_{k}\right\}_{L}=\left\{\Pi_{k}\right\}_{L} \cup \Pi_{k}$.

2. $\max _{\pi \in \Pi k} e_{k}(\pi)>\varepsilon$. The approximation error is higher than the tolerance. We shall split this condition into two further cases, depending on the volume of $\Pi_{k}$ :

a. The volume of $\Pi_{k}$ is smaller than a predefined maximum resolution $\eta$, i.e. $\operatorname{vol}\left(\Pi_{k}\right) \leq \eta$. In these blocks the system nonlinearities are so large as to prevent its LTV approximation within a small volume $\eta$ and thus are not further considered for the subsequent step of the proposed algorithm. Such blocks are left undetermined from the gain tuning standpoint.

b. The volume of $\Pi_{k}$ is higher than $\eta$. $\Pi_{\mathrm{k}}$ is then partitioned into $2^{p}$ sons and the process of evaluating the maximum approximation error is repeated for each of them.

The major challenge in applying the above algorithm resides in the evaluation of the $e_{k}(\cdot)$ function's upper bound over a given $\Pi_{k}$, that is, in determining if the distance between the nonlinear and linear trajectories under the same $\Pi$ is within the tolerance for all $\pi \in \Pi_{k}$, as discussed in the next section.

\subsubsection{Evaluation of nonlinear trajectories approximation error}

A few approaches exist that allow relating the time responses of a nonlinear system to those of its linearization by quantitative means. These approaches conservatively bound from above a certain measure of the trajectories distance by maximizing some nonlinear timevarying test function over a vector space. They thus either solve an optimization problem, with related computational burden, or require prior knowledge of the test function maximum bound, for instance using the Lipschitz constant (Asarin et al., 2007) or the maximum bound of the dynamical function's second order derivatives (Desoer \& 
Vidyasagar, 1975). The latter methods, however, provide bounds on the trajectory distance that are typically exponentially increasing with time. This implies that in practice they can be used for time horizons of limited duration w.r.t. the system time-scales, which is not the case of re-entry applications. Alternative approaches have been proposed, which estimate the approximation error introducing some heuristic methods. In (Rewienski \& White, 2001) the linear system is considered a valid approximation within a norm-ball, whose radius is determined depending on the linear trajectory characteristics. In (Tancredi et al., 2008) the approximation error over a polytope in the parameters space is estimated by its maximum value over the polytope's vertices, assuming that the polytope is sufficiently smaller than the scale at which the system exhibits significant nonlinear behaviour so that the maximum error always occurs in a vertex.

The approximation error is here evaluated in probabilistic terms, as proposed in (Tancredi et al., 2009). In particular, by fictitiously introducing a statistical description of the gains in the generic $\Pi_{k}$, we accept the risk of the approximation error being higher than the tolerance in a subset of $\Pi_{k}$ having small probability measure. More precisely, we consider the nonlinear system to be well approximated in $\Pi_{k}$ if the risk of $e_{k}(\cdot)$ being higher than the error tolerance is smaller than a threshold. The value of this threshold shall be selected sufficiently small as to avoid that $e_{k}(\cdot)$ can be higher than the tolerance with significant probability. However, it shall also be sufficiently high as to avoid that the $\Pi_{k}$ sets have a volume smaller than the maximum resolution $\eta$. Preliminary numerical analyses suggest that in our problem setting a threshold value equal to $6 \%$ is a good compromise:

$$
\Pi_{k}: \operatorname{Pr}\left(e_{k}>\varepsilon\right) \leq 0.06 \Rightarrow \max _{\pi \in \Pi k} e_{k}(\pi) \leq \varepsilon
$$

Without introducing any assumption on the probability distribution of $e_{k}(\cdot)$, we can then use the one-sided Chebyshev inequality to translate Eq.(11) in:

$$
\Pi_{k}: E\left(e_{k}\right)+4 \sqrt{\operatorname{Var}\left(e_{k}\right)} \leq \varepsilon \quad \Rightarrow \quad \max _{\pi \in \Pi k} e_{k}(\pi) \leq \varepsilon
$$

In order to determine the mean and variance of $e_{k}(\cdot)$, we use the Scaled Unscented Transformation (SUT), first introduced in (Julier, 2002). More specifically, let us consider a generic hyper-rectangle $\Pi_{k}:=\left[\underline{\pi}_{1 k}, \bar{\pi}_{1 k}\right] \times \ldots \times\left[\underline{\pi}_{p k}, \bar{\pi}_{p k}\right]$. We fictitiously assume $\pi$ to be uniformly distributed in $\Pi_{k}$, which results in the following mean and covariance matrix, $E_{k}$ and $\operatorname{Cov}_{k}$, respectively.

$$
E_{k}=\pi_{k}^{0} ; \operatorname{Cov}_{k}=\frac{1}{12} \times \operatorname{diag}\left[\left(\bar{\pi}_{k 1}-\underline{\pi}_{k 1}\right)^{2}, \ldots,\left(\bar{\pi}_{k p}-\underline{\pi}_{k p}\right)^{2}\right]
$$

Using the SUT, we may estimate $e_{k}(\cdot)$ mean and variance. Specifically, according to (Julier, 2002), we choose a series of $2 p+1$ points $\Theta_{k i} \in \Pi_{k}$, symmetrically distributed around the mean $\pi_{k}^{0}$, as follows:

$$
\begin{array}{ll}
\Theta_{k i}=\pi_{k}^{0} & i=0 \\
\Theta_{k i}=\pi_{k}^{0}+\left[\sqrt{(p+\chi) \operatorname{Cov}_{k}}\right]_{i} & i=1, \ldots, p \\
\Theta_{k i}=\pi_{k}^{0}-\left[\sqrt{(p+\chi) \operatorname{Cov}_{k}}\right]_{i-p} & i=p+1, \ldots, 2 p
\end{array}
$$


where $\chi=\mu^{2}(p+\kappa)-p$ is a tunable parameter. Each point has an associated weight for computing the mean and variance of the $e_{k}(\cdot)$ function. The first point $\Theta_{k 0}$ has instead two weights, one for computing the mean and one for the variance. Denoting with $W_{i}(m)$ the weights for computation of $\mathrm{E}\left(e_{k}\right)$, and with $W_{i}(c)$ the weights for computation of $\operatorname{Var}\left(e_{k}\right)$, the following hold (Julier, 2002):

$$
\begin{gathered}
W_{0}^{(m)}=\chi /(p+\chi) ; W_{0}^{(c)}=W_{0}^{(m)}+\left(1-\mu^{2}+\beta\right) \\
W_{i}^{(m)}=W_{i}^{(c)}=1 /[2(p+\chi)] \quad i=1, \ldots, 2 p
\end{gathered}
$$

The mean and variance of the $e_{k}(\cdot)$ function can be then estimated as the weighted average and weighted outer product of the transformed points, allowing to evaluate if Eq.(12) holds by numerically evaluating $2 p+1$ times the $e_{k}(\cdot)$ function :

$$
\begin{gathered}
E\left[e_{k}\right]=\sum_{i=0}^{2 p} W_{i}^{(m)} e_{k}\left(\Theta_{k i}\right) \\
\operatorname{Var}\left[e_{k}\right]=\sum_{i=0}^{2 p} W_{i}^{(c)}\left\{E\left[e_{k}\right]-e_{k}\left(\Theta_{k i}\right)\right\}^{2}
\end{gathered}
$$

The SUT has three tunable parameters, $\mu, \beta$, and $\kappa$. Guidelines for tuning these parameters are given by (Van der Merwe et al., 2000), which suggests letting $\kappa=0 . \beta$ is a non-negative weighting term which can be used to incorporate knowledge of the higher order moments of the distribution. Preliminary numerical analyses have shown that in our problem setting $\beta=0$ delivers the best estimates. At last, $\mu$ controls the "size" of the $\Theta$ points distribution and should be $0 \leq \mu \leq 1$. We choose $\mu=(3 / p)^{0.5}$ in order to have the $\Theta_{k i}$ points in the center of $\Pi_{k}$ facets. This choice allows for sharing some computations between adjacent $\Pi_{k}$ sets, and thus to reduce the overall computational load.

\subsection{Property clearance}

Once the $\left\{\Pi_{k}\right\}_{L}$ partition has been determined, one can obtain a solution to problem 1 by formulating a similar problem on the LTV approximating systems corresponding to $\left\{\Pi_{k}\right\}_{L}$. For such LTV systems, the difference between any nonlinear and linear trajectories under the same $\Pi$ is included in the closed ball in $\Re^{n}$ with respect to the norm in Eq. (8), with radius equal to $\varepsilon, B_{\varepsilon}$. It follows that the nonlinear solutions tube is included in the Minkowski sum between the solutions tube of its linearization and the former ball. To exploit this result in achieving the problem's solution, let us define a reduced admissible solution tube, obtained by shrinking $S_{A}(\cdot)$ of an amount equal to $B_{\varepsilon}$. Denoting as $\oplus$ the Minkowski sum operator, the reduced admissible solution tube reads:

$$
S_{A}^{\prime}(t): \quad S_{A}^{\prime}(t) \oplus B_{\varepsilon}=S_{A}(t) \forall t \in[0, T]
$$

The $S_{A}{ }_{A}(\cdot)$ complying to Eq. (17) can be easily determined since $S_{A}(\cdot)$ is hyper-rectangular at each time epoch. Being the norm sphere hyper-rectangular as well by definition, $S_{A}{ }_{A}(\cdot)$ can be obtained simply by component-wise difference of $S_{A}(\cdot)$ and $B_{\varepsilon}$. Consider now a modification of the $P$ property, expressed in terms of $S^{\prime}{ }_{A}(\cdot)$ and of the linear trajectories corresponding to $\left\{\Pi_{k}\right\}_{L}$ : 


$$
P^{\prime}(\pi):= \begin{cases}\text { true } & y_{L k}(t ; \pi) \in S_{A}^{\prime}(t) \forall t \in[0, T] \\ \text { false } & \exists t \in[0, T]: y_{L k}(t ; \pi) \notin S_{A}^{\prime}(t)\end{cases}
$$

It can be easily proved that $P^{\prime}$ implies $P$. Therefore, introducing a region of admissible uncertainties analogous to $\Pi_{A}$, but based on $P^{\prime}$, as $\Pi_{A}^{\prime}:=\left\{\pi \in \Pi \mid P^{\prime}(\pi)=\right.$ true $\}$, it follows that $\Pi_{A}^{\prime} \subseteq \Pi_{A}$. It will be shown later on that a technique exists to obtain a good estimate of $\Pi^{\prime}{ }_{A}$. This is equivalent to obtain a conservative solution to problem 1 , in the sense that the computed region $\Pi_{A}^{\prime}$ will be included in the actual $\Pi_{A}$. Nonetheless, the amount of conservativeness in estimating $\Pi_{A}$ is bounded, and can be reduced as required by reducing the approximation error tolerance, at the price of a higher computational load.

\subsubsection{Computation of $\boldsymbol{\Pi}_{\mathrm{A}}$}

The computation of $\Pi^{\prime}{ }_{A}$ is obtained by exploiting the preservation of convexity in LTV trajectories under constant inputs and by applying a set inversion algorithm, SIVIA (Set Inverter Via Interval Analysis), originally developed in the framework of Interval Analysis. We briefly recall here the algorithm main features relevant to the present context, referring the interested reader to (Jaulin et al., 2001) and the references therein.

Given the definition of $\Pi_{A}^{\prime}$ and $P^{\prime}$, the determination of $\Pi_{A}^{\prime}$ may be seen as a set inversion problem, which is defined as follows. Let $f$ be a possibly nonlinear function from $\Re^{n}$ to $\Re^{m}$, and let $Y$ be a subset of $\mathfrak{R}^{m}$. Set inversion is the determination of the reciprocal image: $X=\left\{x \in \Re^{n} \mid f(x) \in Y\right\}=f^{-1}(Y)$, which in our case is $\Pi^{\prime}{ }_{A}$ itself. The SIVIA algorithm allows to compute two sub-partitions of $\Pi$, that is, partitions of a subset of $\Pi$, that are an inner and outer enclosure of $\Pi_{A}^{\prime}$, denoted as $\underline{\Pi}_{A}^{\prime}$ and $\bar{\Pi}_{A}^{\prime}$ respectively.

$$
\underline{\Pi}_{A}^{\prime} \subset \Pi_{A}^{\prime} \subset \bar{\Pi}_{A}^{\prime}
$$

The algorithm is iterative, and is initially applied to the partition $\left\{\Pi_{k}\right\}_{L}$. In order to determine if a block $\Pi_{\mathrm{k}}$ belongs to the enclosures, it performs an inclusion test $\left[P^{\prime}\right]$, having the following properties:

$$
\begin{aligned}
& {\left[P^{\prime}\right]\left(\Pi_{k}\right)=\text { true } \Rightarrow \forall \pi \in \Pi_{k}, P^{\prime}(\pi)=\text { true }} \\
& {\left[P^{\prime}\right]\left(\Pi_{k}\right)=\text { false } \Rightarrow \forall \pi \in \Pi_{k}, P^{\prime}(\pi)=\text { false }}
\end{aligned}
$$

More precisely, the inner enclosure $\underline{\Pi}_{A}^{\prime}$ is composed of hyper-rectangular blocks $\Pi_{k}$ for which the inclusion test is true. Given Eq. (19), such blocks are also members of $\bar{\Pi}_{A}^{\prime}$. Reversely, if it can be proved that $\left[P^{\prime}\right]\left(\Pi_{k}\right)=$ false, then the block has an empty intersection with $\Pi^{\prime}{ }_{A}$, and it is thus rejected. Otherwise, no conclusion can be drawn based on the inclusion test, and the block $\Pi_{k}$ is called undetermined. The latter is then bisected in $2^{p}$ subsets that are tested until their volume reaches the user-specified resolution $\eta$. Thus, such undetermined minimum-volume blocks are deemed small enough to be stored in the outer approximation $\bar{\Pi}_{A}^{\prime}$ of $\Pi^{\prime}{ }_{A}$. 


\subsubsection{Inclusion test for SIVIA}

The application of SIVIA requires defining an inclusion test, which is typically obtained by applying interval analysis, e.g. in (Juliana et al., 2008). However, interval computation is usually pessimistic, in the sense that a block $\Pi_{k}$ may be deemed undetermined by an inclusion test even if the property under analysis holds uniformly (i.e. attains the same Boolean value) over the block itself. This implies a substantial increase in the computational load, which is particularly critical since the algorithm computational complexity increases exponentially with $p$. In the present context, we use the inclusion test proposed in (Tancredi et al., 2009), which captures exactly the blocks in which $P^{\prime}$ is uniformly true, and also provide a condition which is sufficient for $P^{\prime}$ to be uniformly false.

The inclusion test exploits the preservation of convexity in LTV trajectories under constant inputs. Consider a generic hyper-rectangular $\Pi_{k} \in\left\{\Pi_{k}\right\}_{L}$. $\Pi_{k}$ is a convex polytope having $2^{p}$ vertices $\pi_{k}^{(v)}$, i.e. it admits the following vertex representation:

$$
\Pi_{k}=\left\{\pi \in \mathfrak{R}^{p} \mid \pi=\sum_{v=1}^{2^{p}} \lambda_{k v} \pi_{k}^{(v)}, \lambda_{k v} \geq 0, \sum_{v=1}^{2^{p}} \lambda_{k v}=1\right\}
$$

Because the trajectory of an LTV system under a constant input $\pi$ may be viewed as an affine transformation with respect to $\pi$, any solution of the LTV system under a generic $\pi$ in $\Pi_{k}$ is a convex combination of the solutions under all the $\pi_{k}{ }^{(v)}$. The output trajectories $y_{L k}(t ; \pi)$ thus span the following set (tube) $S_{L k}(t)$ as $\pi$ varies in $\Pi_{k}$.

$$
S_{L k}(t)=\left\{y_{L k}(t ; \pi) \in \mathfrak{R}^{w} \mid y_{L k}(t ; \pi)=\sum_{\nu=1}^{2^{p}} \lambda_{k v} y_{L k}\left(t ; \pi_{k}^{(\nu)}\right), \lambda_{k v} \geq 0, \sum_{\nu=1}^{2^{p}} \lambda_{k v}=1\right\}
$$

As a consequence, the knowledge of the $2^{p}$ vertex trajectories $y_{L k}\left(t ; \pi_{k}(v)\right)$ allows to determine exactly the solutions tube corresponding to $\Pi_{k}$. We exploit this property to define the inclusion test, which is a comparison of the two time varying polytopes $S_{L k}$ and $S_{A}{ }_{A}$. More precisely, the condition $S_{L k}(t) \subseteq S^{\prime}{ }_{A}(t)$ for all $t \in[0, T]$ is equivalent to $P^{\prime}$ being uniformly true in $\Pi_{k}$, and, given $S_{L k}$ convexity, it is equivalent also to the $2^{p}$ vertex trajectories $y_{L k}\left(t ; \pi_{k}(v)\right)$ belonging to $S^{\prime}{ }_{A}(t)$. The condition $S_{L k}(t) \cap S^{\prime}{ }_{A}(t)=\varnothing$ for at least one $t \in[0, T]$ is instead equivalent to $P^{\prime}$ being uniformly false in $\Pi_{k}$. Unfortunately, this condition may not be checked using only the knowledge of the vertex trajectories, but would require further computations to be ascertained exactly. We instead provide a condition involving only the vertex trajectories, which is only sufficient for $S_{L k}(t) \cap S^{\prime}{ }_{A}(t)=\varnothing$. In particular, we exploit the fact that $S^{\prime}{ }_{A}(\cdot)$ is hyper-rectangular by assumption, and thus admits an easily-obtainable half-space representation $S_{A}{ }_{A}(t)=\left\{y \in \mathfrak{R}^{w} \mid S_{A}^{L} y \leq S_{A}^{R}(t)\right\}$, where $S_{A}^{L}=\left(I_{w},-I_{w}\right)^{T}, S_{A}^{R}:[0, T] \rightarrow$ $\mathfrak{R}^{2 w^{\times}} 1$ ( $I_{w}$ stand for the $w$ by $w$ identity matrix). In case at least one of the $2 w$ inequalities defining $S^{\prime}{ }_{A}(\cdot)$ is not satisfied by all the vertex trajectories, the solutions tube $S_{L k}(\cdot)$ lies completely outside $S^{\prime}{ }_{A}(\cdot)$, implying $S_{L k}(t) \cap S_{A}^{\prime}(t)=\varnothing$. We thus define the following inclusion test, which formally resumes the previous discussion. Its evaluation requires a limited (and known a priori) number of linear trajectories, which are obtained by numeric simulation. Note that nonlinear simulations are not needed for the evaluation of the inclusion test, which involves only simulation of the linear approximations. 


$$
\begin{gathered}
{\left[P^{\prime}\right]\left(\Pi_{k}\right):=\text { true } \Leftrightarrow \quad \forall t \in[0, T], \forall v=1, . ., 2^{p}} \\
\\
S_{A}^{L} y_{L k}\left(t ; \pi_{k}^{(v)}\right) \leq S_{A}^{R}(t)
\end{gathered}
$$

Applying the above procedure, $\Pi_{A}^{\prime}$ is determined exactly within a prefixed resolution, and, due to the properties of the LTV systems defined on $\left\{\Pi_{k}\right\}_{L}$, problem 1 is solved conservatively for the nonlinear system (1).

\section{Application case}

This section introduces the nonlinear system describing the closed-loop longitudinal flight dynamics of the experimental reusable launch vehicle demonstrator USV-FTB1 currently operated by the CIRA. The FTB1 vehicle is the first of three planned vehicle configurations that CIRA is developing as part of its USV Program, whose main goal is contributing to the international community effort toward the development of next generation reusable space vehicles. This vehicle is planned to execute flight tests in subsonic, transonic and low supersonic flight regimes, in view of the development of upgraded vehicle configurations to perform sub-orbital and orbital re-entry flights. The FTB1 vehicle, described in detail in (Russo, 2009) and shown in Fig. 1, is unmanned and un-powered. It has a slender wing configuration, with two sets of aerodynamic effectors: the elevons, which provide pitch control when deflected symmetrically and roll control when deflected asymmetrically, and the rudders for yaw control.

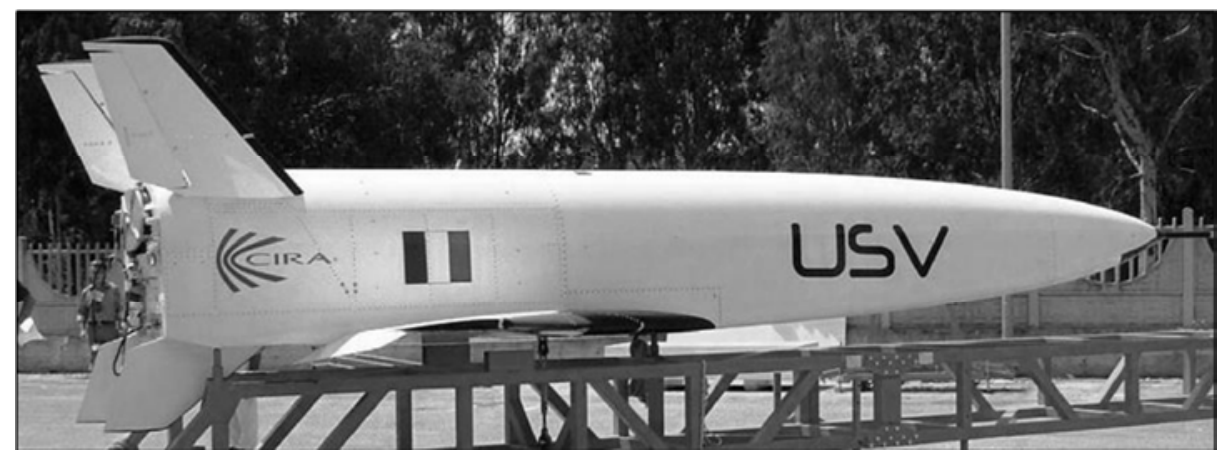

Fig. 1. USV-FTB1 vehicle.

The second Dropped Transonic Flight Test (DTFT-2) of the FTB1 vehicle is specifically considered to show the effectiveness of the proposed technique. The mission, successfully executed in April 2010, foresees a drop of the vehicle from a stratospheric balloon (at nearly null velocity and angle of attack) to reach Mach numbers in the range of [1.2 $\div 1.3]$ for investigating aerodynamics and advanced guidance navigation and control in the transonic phase of an un-powered re-entry flight. The basic operations of the mission consist of an 
ascent phase during which the stratospheric balloon brings the FTB1 at the release altitude of about at about $24-26 \mathrm{~km}$ followed by a flight phase where the FTB1 is dropped and the aerodynamic controlled flight starts. The vehicle accelerates until the desired Mach number is reached, and then starts a Mach-hold phase in which it performs a sweep in angle of attack for maintaining a constant Mach number. A deceleration phase is then initiated (up to $0.2 \mathrm{Mach}$ ) at the end of which a recovery parachute is deployed. The mission ends with the demonstrator splash down in the Mediterranean Sea.

Because the scope of the present section is to demonstrate the effectiveness of the gain tuning technique on an application of practical engineering relevance, we will restrict the analysis to a simplified version of the longitudinal FCL of the FTB1 vehicle, which was used in the initial design phases for executing flight mechanics analyses. Note that the FCL analyzed in this section is significantly different from the ones implemented for the DTFT2 mission (see, for instance, Morani et al., 2011, for a detailed description of the guidance law). Given the nonlinear augmented longitudinal dynamics of the FTB1 vehicle in the DTFT2 mission, the aim of the present analysis is to find (a set of) the controller's gains compliant to a requirement expressed as inclusion in a solution's tube.

The FTB1 vehicle longitudinal dynamics are modelled by means of standard nonlinear equations (Etkin \& Reid, 1996), yielding a sixth order model. Actuator dynamics are included by means of a second order system and first order filters are used for modelling the navigation sensors for $a$ and $q$. The longitudinal dynamics are augmented by a proportional-derivative flight control law, arranged in a cascade structure with feedback on the pitch rate $q$ and angle of attack $a$. The augmented vehicle is driven by a time-varying angle of attack reference signal $a_{\text {ref, }}$ which ramps up from zero at the vehicle release from the stratospheric balloon up to $8 \mathrm{deg}$. in the initial drop phase. The angle of attack is held constant until the desired Mach number of about 1.2 is reached. The Mach hold phase follows, where an a - sweep manoeuvre is performed. At the end of the Mach hold phase, the angle of attack increases up to 10 deg., value maintained in low subsonic conditions until parachute deployment. The overall feedback action is shown in Fig. 2 and has the following analytical expression, where $\zeta$ stands for the FCL internal state.

$$
\delta_{e}=k_{3}\left[k_{1}\left(\alpha_{r e f}-\alpha\right)+\zeta-q\right] ; \dot{\zeta}=k_{1} k_{2}\left(\alpha_{r e f}-\alpha\right)
$$

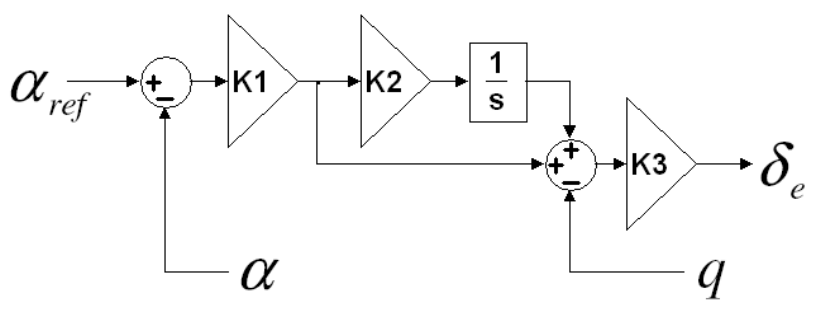

Fig. 2. FCL structure.

Two of the three gains of the controller, $k_{1}, k_{2}$, and $k_{3}$ are scheduled depending on the dynamic pressure $q_{\infty}$, according to $k_{1}=k_{10}, k_{2}=k_{20}+k_{2 s} q_{\infty}$, and $k_{3}=k_{30}+k_{3 s} / q_{\infty}$. The FCL foresees thus five gains. The nominal gains setting is $k_{1}^{\prime}=1.25 \mathrm{~s}^{-1} ; k_{20}^{\prime}=-1.28 \mathrm{~s}^{-1}$; 
$k^{\prime}{ }_{2 s}=4.48 \cdot 10^{-4} \mathrm{~m} \cdot \mathrm{s} \cdot \mathrm{kg}^{-1} ; k^{\prime}{ }_{30}=1.73 \cdot 10^{-1} \mathrm{~s}_{;} k^{\prime}{ }_{3 s}=-7.76 \cdot 10^{3} \mathrm{~kg} \mathrm{~s}{ }^{-1} \cdot \mathrm{m}^{-1}$. These gains have been determined applying standard LTI control synthesis techniques, and the resulting control law yields satisfactory LTI stability characteristics. Because of the complexity of the LTI based analysis when applied to these vehicles flying markedly time-varying trajectories, and because the focus of the present chapter is on determining the effectiveness of the proposed technique in dealing with time-based control performance requirements, the results of the stability analysis are not shown here for brevity. The reader is referred to (Tancredi et al., 2011) for an overview of the LTI stability analysis in a similar application.

The nominal response is obtained applying the above gain tuning, and considering the system to start at $t_{0}=21.55 \mathrm{~s}$. This is the first time epoch at which the Mach number is at least equal to 0.7 , i.e. $M \geq 0.7$, which is the threshold condition above which the actuation system gains sufficient command authority for controlling the angle of attack.

The nominal response's angle of attack and commanded elevon deflections $\delta_{e}$ are shown in Fig. 3. The initial oscillation in $a$ is caused by a sharp decrease of the elevons efficiency in the transonic phase. However, because of the considerable uncertainty on the entity of this phenomenon, no dedicated feed-forward actions were implemented.
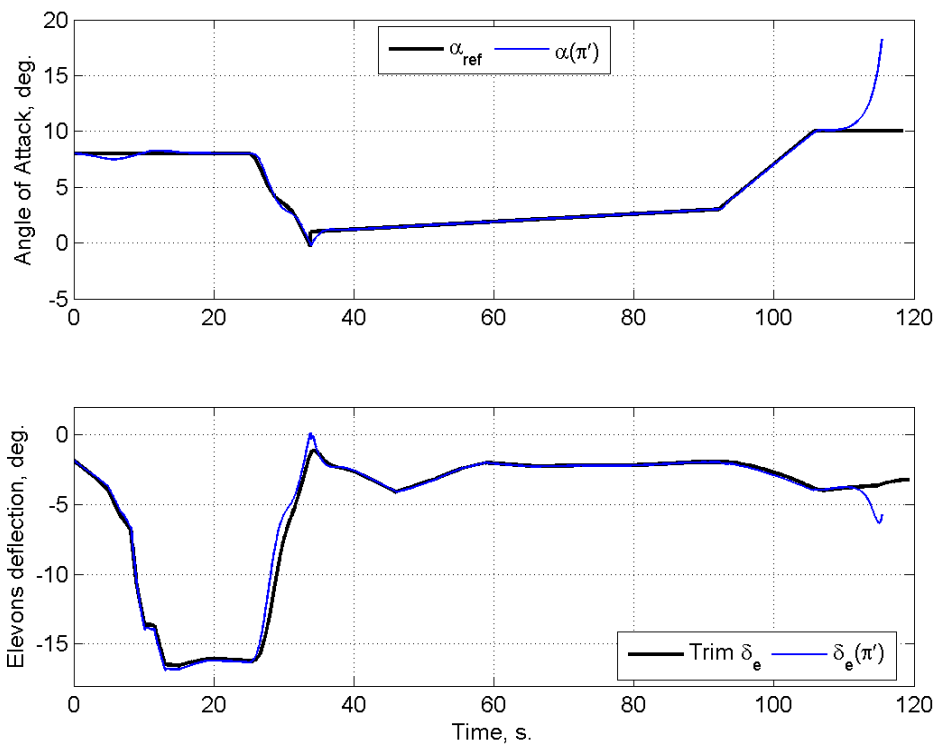

Fig. 3. Nominal response time histories.

The tuning analysis has been performed on a subset of the FCL gains. More precisely, we analyzed effects on the variations of the three most influential gains: the proportional a constant gain $k_{1}$, the scheduling gain of the integral a action $k_{2 s}$ and the scheduling gain of the proportional q action $k_{3 s}$. In other words, we analyze the effects of the three dimensional vector $\pi:=\left(\begin{array}{llll}k_{1} & k_{2 s} & k_{3 s}\end{array}\right)^{\mathrm{T}}$, in the following hyper-rectangular domain $K$, centred about the nominal tuning $\pi^{\prime}$. 


$$
\Pi:=\left[0,2 k_{1}^{\prime}\right] \times\left[0,2 k_{2 s}^{\prime}\right] \times\left[2 k_{3 s}^{\prime}, 0\right]
$$

The maximum allowed distances of the above variables for a meaningful linearization are set to $0.2 \mathrm{deg}$ in angle of attack, $1.8 \mathrm{deg} \cdot \mathrm{s}^{-1}$ in pitch rate and $1.5 \mathrm{deg}$ in elevons deflection. The admissible solutions tube constrains only the angle of attack and the elevons deflections. Elevons deflection are required to be within $[-20,20]$ deg., which represent the limits of the actuation system. The solutions tube in a is tailored around the reference signal $a_{\text {ref, }}$ enforcing the required maximum tracking error of $\pm 0.6 \mathrm{deg}$. Because of the previously mentioned oscillation, the tracking requirement is relaxed to $\pm 0.8 \mathrm{deg}$. in the transonic phase. The final a hold phase is treated separately from the remainder of the trajectory. Indeed, both tracking requirements are less stringent in this phase, increasing up to \pm 2 deg., and the vehicle flight performances are dramatically different in these low subsonic flight conditions than in the remainder of the trajectory. Separating the tracking requirements in these two parts of the trajectory allows for a clearer understanding of the method potentials. Because of this setting, two admissible solution tubes are introduced: the final tube, which enforces requirements only on the final a-hold phase, and the tracking tube, which enforces tracking requirements in the remainder of the trajectory. Note that since the linearization error is taken into account in the admissible solutions tube definition (see section 3.2), the $a_{\text {ref }}$ tracking requirements to which the linearized solutions shall comply are tighter than the enforced ones of $\pm 0.2 \mathrm{deg}$. Fig. 4 shows the two required solutions tubes in a, as well as the above mentioned "reduced" bounds. Note that the nominal tuning does not comply with any of the two tubes.
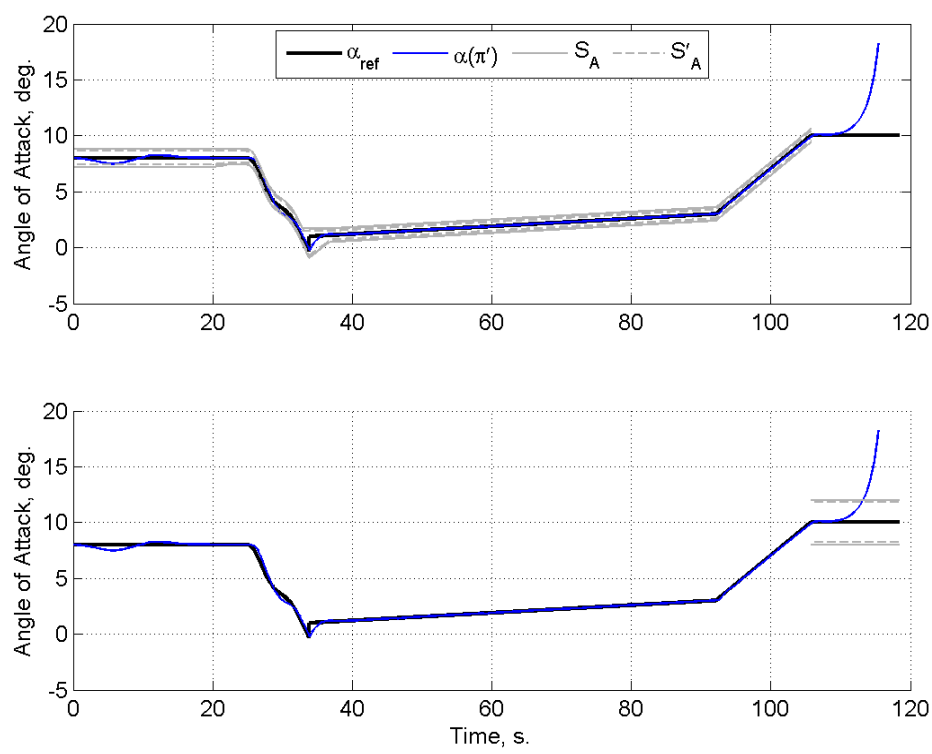

Fig. 4. Admissible solution tubes. Tracking (top) and Final (bottom). 


\subsection{Results}

The approximation phase results are collected in Fig. 5, showing $\left\{\Pi_{k}\right\}_{L}$, the partition into which the gain domain $\Pi$ has been divided to obtain a meaningful linearization. Results show that the original nonlinear system is successfully approximated only in a subset of the gain domain. In the remainder of $\Pi$, the system state vector dependency on the gains is highly nonlinear, and prevents the system to be approximated by its time-varying linearization even in $\Pi_{k}$ subsets with the minimum allowed volume $\eta$ (see section 3.1). The approximation phase results were obtained with a CPU time of $\sim 10$ hours on a standard personal computer. Note however, that its results do not depend on the admissible solutions tube, and are thus used for both the tracking and the final ones without the need of computing the approximation twice. The property clearance phase calls for a computational load that is only a fraction of the approximation one. In fact, evaluation of the inclusion test in Eq.(23) needs the numerical simulation only of the linear approximating systems, and nonlinear simulations are not involved at all. Fig. 6 collects the clearance phase results for the tracking tube. It can be seen how the inclusion test of Eq.(23) divides the blocks of the partition $\left\{\Pi_{k}\right\}_{L}$. The property clearance phase builds upon simulation of the linear approximations, and thus requires only about 1 hour of computation time. The region in which the gains comply with the tracking requirements, $\Pi^{\prime}{ }_{A}$, is shown in Fig. 7 for both the tracking and final tubes. As anticipated, the compliance region of both tubes does not comprise the nominal tuning. Compliance to each of the two tubes calls for lower than nominal values of the scheduling gain of the proportional q action, $k_{3 s}$, coupled to higher proportional and integral scheduling gains of $\mathrm{a}, k_{1}$ and $k_{2 s}$, respectively. Requirements yielding to the final tube, however, are much more restrictive than those in the remainder of the trajectory, as can be seen by the small dimensions of the corresponding $\Pi_{A}^{\prime}$ region.

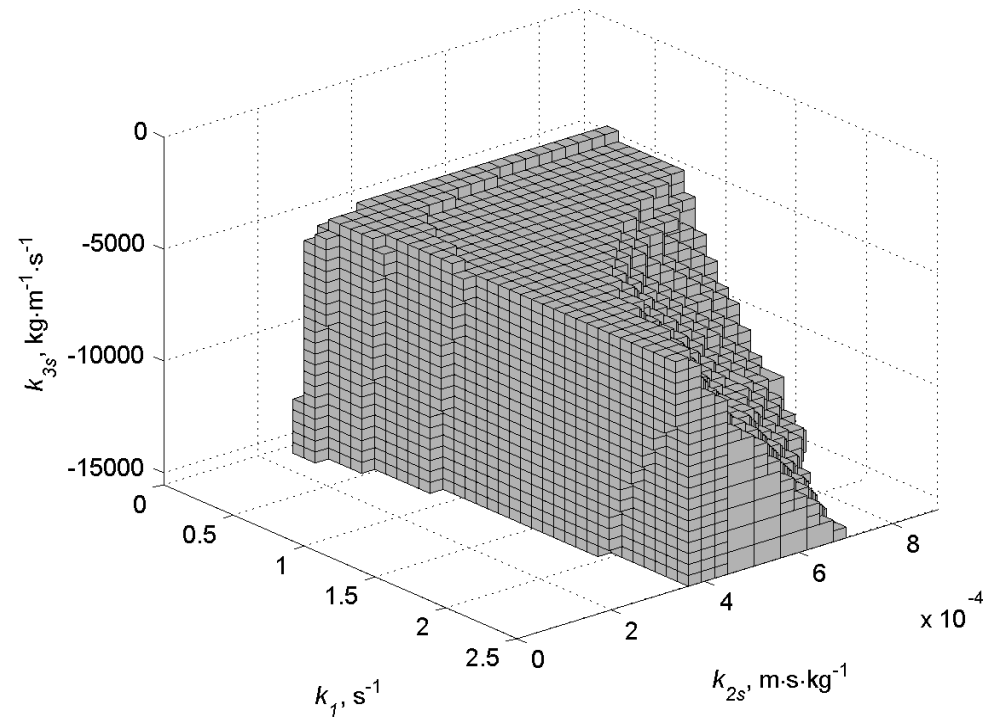

Fig. 5. Approximation results: $\left\{\Pi_{k}\right\}_{L}$ 


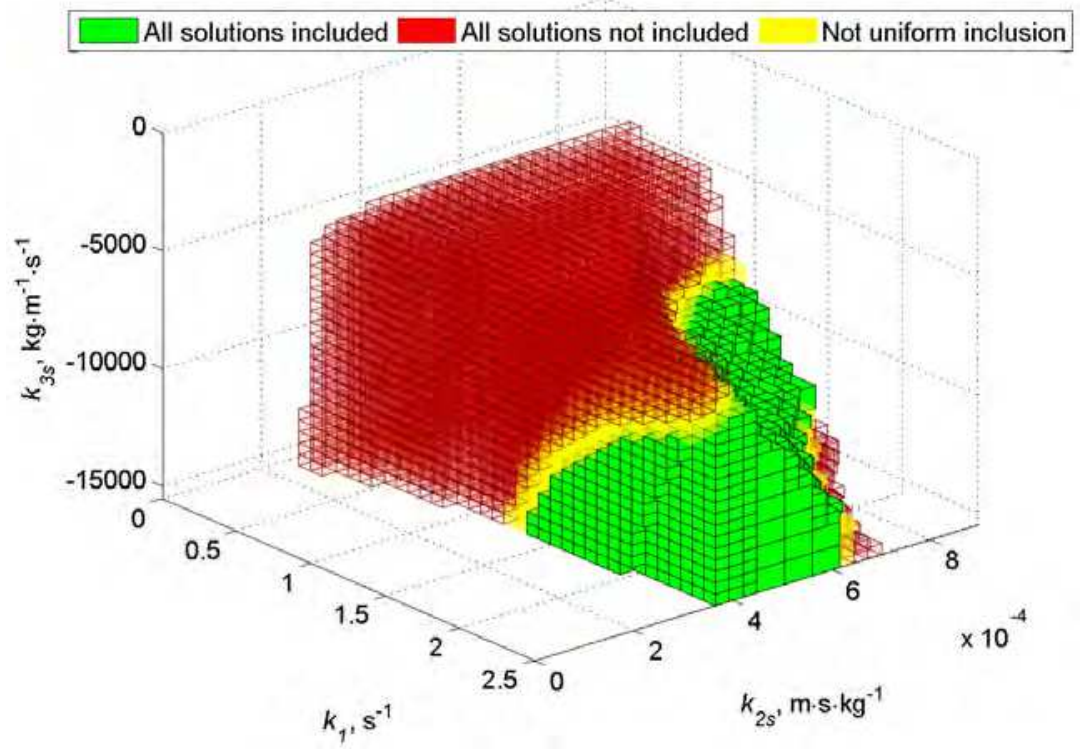

Fig. 6. Property Clearance results - Tracking tube.

These results demonstrate one of the main advantages of the proposed approach, that is, the capability to support the physical understanding of all the causes for unsatisfactory performances of the FCL within the whole $\Pi$ region, being confident of having covered all possible gain combinations of interest. Fig. 8 compares the compliance regions of the two tubes, which are disjoint by a very small offset. However, because the offset dimensions are comparable to the resolution at which the results have been obtained, the true compliance region of the tracking tube may extend as to intersect the final tube's one. Even if this may in principle also not be the case, common sense suggests that a tuning lying near this offset would have tracking performances that do not violate significantly both tubes.

At last, we present the nonlinear system's simulation for a candidate tuning. In order to select this "optimal" tuning, $\Pi_{\mathrm{opt}}$, we choose the root mean square (RMS) of the $a_{\text {ref }}$ tracking error as a cost function. The so obtained optimal tuning is shown in Fig. 8 as well, and is compared to the nominal one in Table 1 and in Fig. 9. Results show that the optimal gain yields a significantly smaller RMS error in tracking $a_{\text {ref }}$ than the nominal one, and improves the system behaviour in the final phase.

\begin{tabular}{ccccc}
\hline Gains & $k_{1}, \mathrm{~s}^{-1}$ & $; k_{2 s}, \mathrm{~m} \cdot \mathrm{s} \mathrm{kg}^{-1}$ & $; k_{3 s}, \mathrm{~kg} \mathrm{~s}^{-1} \mathrm{~m}^{-1}$. & RMS error, deg \\
\hline$\pi^{\prime}$ & 1.25 & $4.48 \cdot 10^{-4}$ & $-7.76 \cdot 10^{3}$ & 0.34 \\
\hline$\pi_{\text {opt }}$ & 1.81 & $3.21 \cdot 10^{-4}$ & $-8.54 \cdot 10^{3}$ & 0.15 \\
\hline
\end{tabular}

Table 1. Comparison of nominal and optimal gains. 

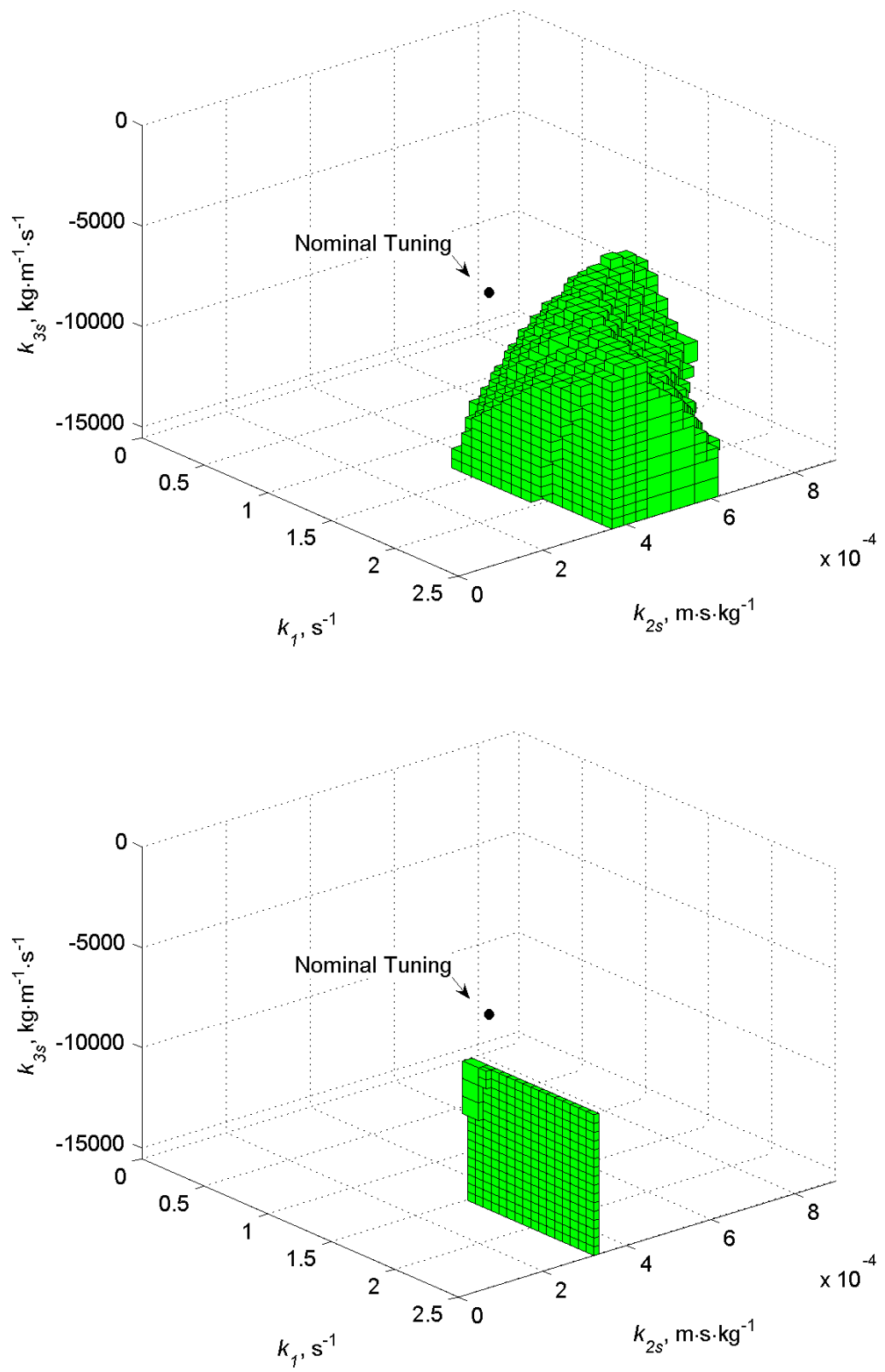

Fig. 7. Nominal tuning vs. compliance region $\Pi^{\prime}{ }_{A}$. Tracking tube (top) and Final Tube (bottom) 


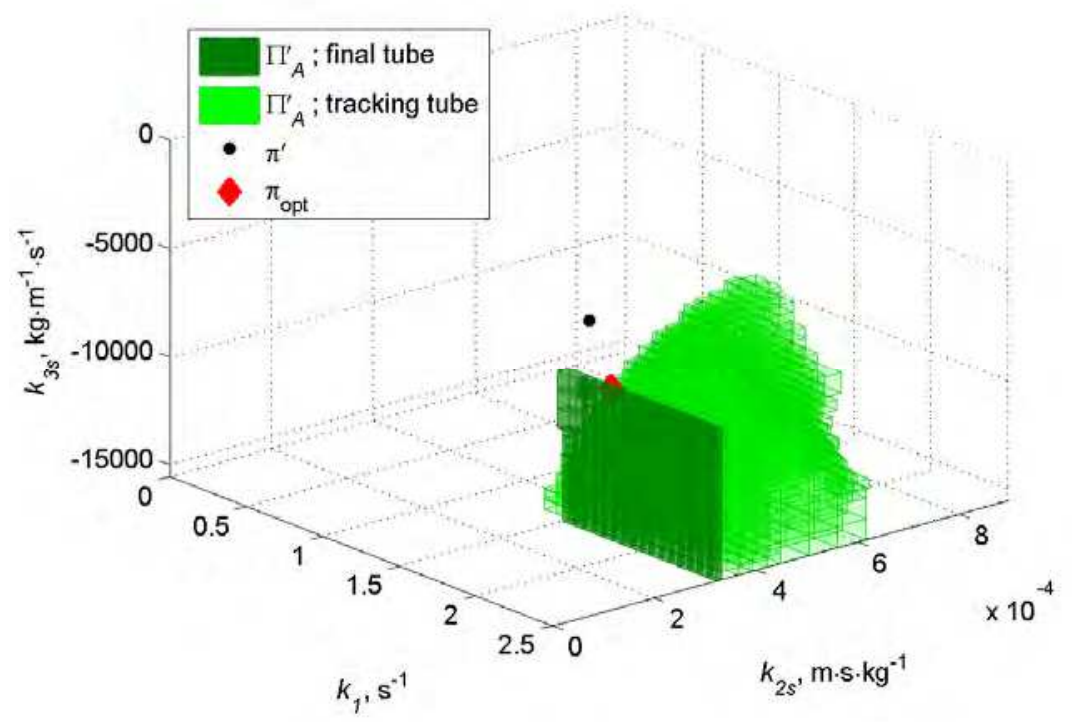

Fig. 8. Comparison between tracking and final tubes compliance regions.

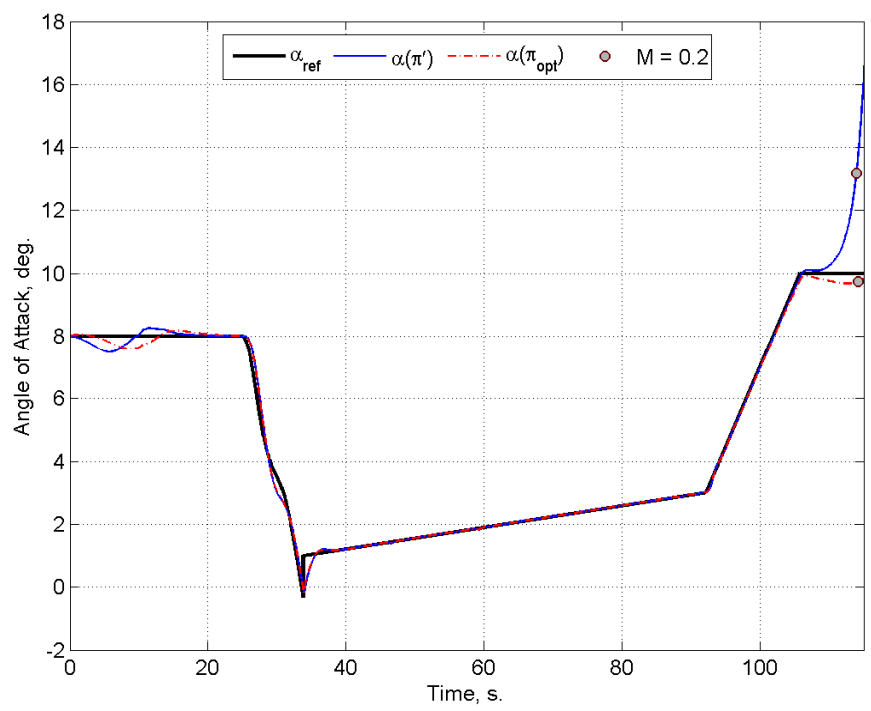

Fig. 9. Selected gains nonlinear simulations: a time history. 


\section{Conclusion}

A novel approach to gain tuning has been developed, based on previous results that were obtained by the authors for a different problem. The approach specifically applies to gliding vehicles in the terminal phases of re-entry flight, and is capable of handling gain scheduled control laws under trajectory tracking requirements. Its capability of highlighting the causes for requirement violation, being confident of having covered all possible combinations of the controller gains, makes the developed technique an effective tool for driving the control law refinement, as shown in an application of practical engineering significance. The adoption of practical stability as a criterion for enforcing trajectory tracking requirements is promising thanks to its inherent capability of handling the original mission or system requirements. In fact, it allows taking explicitly into account trajectory time-varying effects in the tuning task, which can be significant for the applications of interest. The practical stability approach improves the accuracy in evaluating the control law performances with respect to frozen-time approaches, thus reducing the risk of highlighting effects that were not previously disclosed when applying numerical verification methods, such as Monte Carlo techniques. This would avoid the need of upgrading a control design tuning with scarce information on the causes for unsatisfactory performance, as it typically occurs when applying numerical verification methods early in the design cycle, thus streamlining the overall design cycle. In this sense, the proposed approach is though to be complementary both to classical LTI-based design tools and to numerical verification methods.

One important issue of the method is in the number of gains that can be simultaneously treated, due to the exponential increase in the computational load. Nonetheless, its application so far suggests that, when the method is executed on a standard desktop computer, the maximum dimension of manageable problems is in the order of five, depending on the features of the specific application case, most notably its nonlinearity in the whole uncertainty domain. For the application shown in the chapter, the map relating the system state vector to gain values was determined to be heavily nonlinear. This feature is thought to be distinctive of most gain tuning problems, as suggested by common sense and relevant literature, even though further investigations would be needed for ascertaining this claim. This pronounced nonlinearity further limits the method applicability because accurate linear approximations are valid only in small subsets of the gain domain, thus calling for a refined partition, which causes an increase in the computational load. Nonetheless, distributed computing and the use of more powerful computing machines substantially increase the number of gains that can be taken into account.

At last, the presented approach is based on the practical stability criterion, which allows translating tracking requirements in terms of the maximum tracking error. However, in most trajectory tracking applications, the RMS tracking error is also included in the requirements. Note that the RMS error is a convex function of the tracked variable. As such, defining an opportune Boolean property being true when the RMS error is below a certain threshold, one should be capable of devising an inclusion test similar to the one presented in this chapter. This would allow extending the approach for being capable of handling requirements on both maximum and RMS tracking errors. Further work will concern this possibility. 


\section{References}

Amato F., Ariola, M., Cosentino, C., Abdallah, C.T., and Dorato, P. (2003). Necessary and Sufficient Conditions for Finite-Time Stability of Linear Systems, Proceedings of the 2003 American Control Conference, Vol. 5, pp. 4452- 4456.

Asarin, E., Dang, T., and Girard, A. (2007). Hybridization methods for the analysis of nonlinear systems, Acta Informatica, Vol.43, no. 7, pp. 451-476.

Crespo L. G., Kenny S. P., Giesy D. P. (2008). A Verification-driven Approach to Control Analysis and Tuning, Proceedings of the AIAA Guidance Navigation and Control Conference, Honolulu, Hawaii, AIAA-2008-6340.

Crespo, L. G., Matsutani, M., Annaswamy A. M. (2010). Verification and Tuning of an Adaptive Controller for an Unmanned Air Vehicle, Proceedings of the AIAA Guidance Navigation and Control Conference. Toronto, Ontario, Canada, AIAA-2010-8403.

Desoer, C. A., and Vidyasagar, M. (1975). Feedback systems: input-output properties, Academic Press, Inc., New York, Chap. 4.

Dorato, P. (2006). An Overview of Finite-Time Stability, Current Trends in Nonlinear Systems and Control: In Honor of Petar Kokotovic and Turi Nicosia, , Birkhauser Boston, pp 185-195.

dos Santos Coelho, L. (2009). Tuning of PID controller for an automatic regulator voltage system using chaotic optimization approach, Chaos, Solitons \& Fractals, Volume 39, Issue 4, Pages 1504-1514, ISSN 0960-0779.

Etkin, B., and Reid, L.D. (1996). Dynamics of Flight: Stability and Control, John Wiley and Sons, Inc, New York, 3rd ed.

Gruyitch, L., Richard, J-P., Borne P., and Gentina, J.C. (2000). Stability Domains, Chapman \& Hall/CRC, Boca Raton, FL, Chaps. 1,2, 6.

Jaulin, L., Kieffer, M., Didrit, O., Walter, E. (2001). Applied Interval Analysis, with Examples in Parameter and State Estimation, Robust Control and Robotics, Springer-Verlag, London, Chaps. 2, 3.

Juliana, S., Chu, Q.P., Mulder, J.A. (2008). Reentry Flight Clearance Using Interval Analysis, AIAA Journal of Guidance, Control, and Dynamics, Vol.31, n.5, pp.1295-1307.

Julier, S. J. (2002). The scaled unscented transformation, Proceedings of the 2002 American Control Conference, Vol.6, pp. 4555-4559.

Lee, H. C., and Choi, J. W. (2004). Linear Time-Varying Eigenstructure Assignment with Flight Control Application. IEEE Transactions on Aerospace and Electronic Systems, Vol. 40, No. 1, pp. 145- 157.

Leith, D. J., and Leithead, W. E. (2000). Survey of gain-scheduling analysis and design, International Journal of Control, Vol. 73, No. 11, pp. 1001-1025.

Miyazawa Y., Motoda, T. (2001). Stochastic Parameter Tuning Applied to Space Vehicle Flight Control Design, AIAA Journal of Guidance, Control and Dynamics, Vol. 24, No. 3, pp. 597-604.

Morani, G, Cuciniello, G, Corraro, F, and Di Vito, V. (2011). On-line guidance with trajectory constraints for terminal area energy management of re-entry vehicles, Proceedings of the Institution of Mechanical Engineers, Part G: Journal of Aerospace Engineering, 225: 631.

Rewienski, M., White, J. (2001). A Trajectory Piecewise - Linear Approach to Model Order Reduction and Fast Simulation of Nonlinear Circuits and Micromachined Devices, Proceedings of the 2001 IEEE/ACM international conference on Computer-aided design, pp. $252-257$. 
Russo, G. (2009). DTFT-1: Analysis of the first USV flight test, Acta Astronautica, Volume 65, Issues 9-10, Pages 1196-1207, ISSN 0094-5765.

Ryali, V., Moudgalya, K.M. (2005). Practical stability analysis of uncertain nonlinear systems, Proceedings of the National Conference on Control and Dynamic Systems, I.I.T. Bombay.

Tancredi, U., Grassi, M., Corraro, F., Filippone, E., and Russo, M. (2008). A Novel Approach to Clearance of Flight Control Laws over Time Varying Trajectories", Automatic Control in Aerospace, Vol. 1, No. 1, Paper 2, Retrieved from:

<http://www.aerospace.unibo.it/index.php?e=5>

Tancredi, U., Grassi, M., Corraro, F., and Filippone E. (2009). Robustness Analysis for Terminal Phases of Reentry Flight, AIAA Journal of Guidance, Control, and Dynamics, Vol. 32, No. 5, pp. 1679 - 1683.

Tancredi, U., Grassi, M., Corraro, F., Vitale, A., \& Filippone, E. (2011). A linear time varying approach for robustness analyses of a re-entry flight technology demonstrator, Proceedings of the Institution of Mechanical Engineers, Part G: Journal of Aerospace Engineering, in press.

Van der Merwe, R., De Freitas, N., Doucet, A., and Wan, E. A. (2000). The Unscented Particle Filter, Advances in Neural Information Processing Systems, pp.584-590.

Wang, Q., and Stengel, R. F. (2002). Robust control of nonlinear systems with parametric uncertainty, Automatica, Vol. 38, No. 9, pp. 1591-1599. 


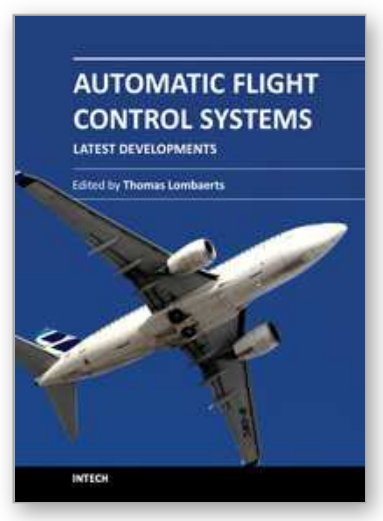

\author{
Automatic Flight Control Systems - Latest Developments \\ Edited by Dr. Thomas Lombaerts
}

ISBN 978-953-307-816-8

Hard cover, 204 pages

Publisher InTech

Published online 18, January, 2012

Published in print edition January, 2012

The history of flight control is inseparably linked to the history of aviation itself. Since the early days, the concept of automatic flight control systems has evolved from mechanical control systems to highly advanced automatic fly-by-wire flight control systems which can be found nowadays in military jets and civil airliners. Even today, many research efforts are made for the further development of these flight control systems in various aspects. Recent new developments in this field focus on a wealth of different aspects. This book focuses on a selection of key research areas, such as inertial navigation, control of unmanned aircraft and helicopters, trajectory control of an unmanned space re-entry vehicle, aeroservoelastic control, adaptive flight control, and fault tolerant flight control. This book consists of two major sections. The first section focuses on a literature review and some recent theoretical developments in flight control systems. The second section discusses some concepts of adaptive and fault-tolerant flight control systems. Each technique discussed in this book is illustrated by a relevant example.

\title{
How to reference
}

In order to correctly reference this scholarly work, feel free to copy and paste the following:

Urbano Tancredi and Federico Corraro (2012). Gain Tuning of Flight Control Laws for Satisfying Trajectory Tracking Requirements, Automatic Flight Control Systems - Latest Developments, Dr. Thomas Lombaerts (Ed.), ISBN: 978-953-307-816-8, InTech, Available from: http://www.intechopen.com/books/automatic-flightcontrol-systems-latest-developments/gain-tuning-of-flight-control-laws-for-satisfying-trajectory-trackingrequirements

\section{INTECH}

open science | open minds

\section{InTech Europe}

University Campus STeP Ri

Slavka Krautzeka 83/A

51000 Rijeka, Croatia

Phone: +385 (51) 770447

Fax: +385 (51) 686166

www.intechopen.com

\section{InTech China}

Unit 405, Office Block, Hotel Equatorial Shanghai

No.65, Yan An Road (West), Shanghai, 200040, China 中国上海市延安西路65号上海国际贵都大饭店办公楼405单元

Phone: +86-21-62489820

Fax: $+86-21-62489821$ 
(C) 2012 The Author(s). Licensee IntechOpen. This is an open access article distributed under the terms of the Creative Commons Attribution 3.0 License, which permits unrestricted use, distribution, and reproduction in any medium, provided the original work is properly cited. 